\title{
Diclofenac prevents temporal increase of intraocular pressure after uneventful cataract surgery with longer operation time
}

\author{
Masahiko Shimura' \\ Toru Nakazawa ${ }^{2}$ \\ Kanako Yasuda' \\ Takashi Shiono 3 \\ Kohji Nishida ${ }^{2}$ \\ 'Department of Ophthalmology, \\ NTT East Japan Tohoku Hospital, \\ Sendai, Japan; ${ }^{2}$ Department \\ of Ophthalmology and Visual \\ Science, Tohoku University Graduate \\ School of Medicine, Sendai, Japan; \\ ${ }^{3}$ Department of Ophthalmology, \\ Shiono Eye Center, Sendai, Japan
}

\begin{abstract}
Purpose: This study compares the effect of topical diclofenac with that of betamethasone against postoperative increase of intraocular pressure (IOP) after cataract surgery in normal patients, and also investigated the risk factors for postoperative increase of IOP in each group.

Methods: Fifty consecutive patients without systemic disease who have bilateral and symmetrical cataracts underwent uncomplicated cataract surgery in both eyes (100 eyes in total). Postoperatively, topical diclofenac was applied 4 times daily to one eye, and topical betamethasone to the other eye in each patient. IOP and best corrected logMAR visual acuity (BCVA) in each eye were measured up to 8 weeks. Total surgery time and effective phacoemulsification time (EPT) for each case was recorded.

Results: BCVA in both diclofenac- and betamethasone-treated eyes significantly improved after the cataract surgery; however, no statistical difference in VA was noted between the diclofenac- and betamethasone-treated eyes throughout the observation period. IOP in the diclofenac-treated eyes decreased with time, in contrast to the IOP in the betamethasone-treated eyes, which showed a slight increase. At 4 and 8 weeks postoperatively, there was significant difference between these two eye groups. Multiple regression analysis revealed that postoperative increase in IOP at 8 weeks in the betamethasone-treated eyes was closely correlated with total surgery time and EPT, but the IOP in the diclofenac-treated eyes showed no correlation with any surgical or clinical parameters.

Conclusions: Postoperative increase in IOP after cataract surgery was affected by total surgery time and EPT in the betamethasone-treated eye. The time for surgery and EPT is longer in complicated cases including patients with a hard nucleus or small pupils, and also longer for beginning surgeons and in older patients. In these cases, diclofenac in place of betamethasone as a postoperative topical antiinflammatory drug is recommended for the prevention of postoperative increase in IOP.
\end{abstract}

Keywords: intraocular pressure, cataracts, visual acuity, betamethasone, diclofenac

Modern cataract surgery uses a small incision and a shorter operation time; therefore, postoperative inflammation is rarely seen. Nevertheless, cataract surgery for severe cases, including cataract grade greater than Emery classification level IV, sometimes takes longer time for emulsification, and causes post-operative inflammation and a subsequent increase in intraocular pressure (IOP), which causes possible neuronal damage. Thus, to reduce the risk of inflammation after cataract surgery, a topical antiinflammatory drug application is widely used. ${ }^{1,2}$ Two classes of compounds are available: corticosteroids and nonsteroidal antiinflammatory drugs (NSAIDs). Although both drugs are effective in suppressing postoperative inflammation, corticosteroids have been reported to increase IOP. ${ }^{3,4}$ Diclofenac is a NSAID which 
inhibits prostaglandin synthesis, ${ }^{5}$ and previous studies have assessed the effectiveness of diclofenac in treating ocular inflammation..$^{6-8}$ Although it has been reported that diclofenac does not cause postoperative increase of IOP, ${ }^{9}$ case-control studies have not been perfomed. A case-control study should be conducted in patients with eligible eyes, where one eye was treated with betamethasone and the other eye with diclofenac. These cases should be conducted in patients without systemic conditions.

In this paper, a prospective case-control compared and evaluated eyes treated with topical corticosteroids and those treated with topical NSAIDs for postoperative increase in IOP after cataract surgery. Also, the relationship between postoperative IOP and clinical factors, including age, gender, preoperative IOP, total surgery time, sonication time, and sonication power was investigated using multiple regression analysis. The final goal of this study was to determine which cases are at risk for postoperative increase in IOP and should be treated with topical administration of NSAIDs.

\section{Methods}

\section{Patient eligibility}

In this prospective study, patients had uncomplicated senile cataracts in both eyes from grade III to IV based on Emery's classification. ${ }^{10}$ Exclusion criteria were ocular hypertension (IOP > $21 \mathrm{mmHg}$ ), glaucoma requiring ocular medication, pseudoexfoliation, uveitis, an endothelial cell count less than 1500 cells $/ \mathrm{mm}^{2}$, corneal opacity, high myopia, previous ocular surgery, systemic disease affecting the inflammatory response such as diabetes mellitus, collagen disease requiring long-term systemic steroids or antiinflammatory drugs, and severe hypertension. Patients whose cataract grades were not symmetrical in both eyes were also excluded in this study. Intraoperative exclusion criteria were iatrogenic iris damage, retained cortical matter, and a serious complication such as posterior capsule rupture. 50 consecutive patients ( 21 male; 29 female) participated in this study.

All patients received a comprehensive ocular examination before treatment and were followed for periodic examinations after the treatments. After informing the patient of the purpose of this study and the possible outcomes, informed consent was obtained from all patients prior to the intervention. This study was also approved by the Ethics Committee for Clinical Research at NTT East Japan Tohoku Hospital using procedures which conformed to the tenets of the Declaration of Helsinki.

\section{Study design}

All patients had cataract surgery performed in both eyes within a 1 week interval. Before cataract surgery, a topical antimicrobial drug, gatifloxacin hydrate $0.3 \%$ ophthalmic solution (Gatiflo ${ }^{\circledR}$; Senju Pharmaceutical Co. Ltd., Osaka, Japan), was administered 4 times/day in both eyes of each patient. The surgical technique used in this study consisted of a continuous curvilinear capsulorrhexis (CCC), phacoemulsification, and implantation of a foldable acrylic IOL (Acryfold ${ }^{\circledR}$ YA-65BB; HOYA medicals, Tokyo, Japan) into the lens capsule through a $3.0 \mathrm{~mm}$ corneoscleral incision. Phacoemulsification was done with an Alcon Legacy $20000^{\mathrm{TM}}$ unit with standard cassettes and tubing. The incision was not sutured. In each patient, cataract surgery in the eye with the worst visual acuity was done first, then surgery in the other eye was completed within a week. If the visual acuity in both eyes was the same, then the right eye was chosen first. The effect of $0.1 \%$ diclofenac (Diclod ${ }^{\circledR}$; Wakamoto Pharmaceutical Co. Ltd., Tokyo, Japan), which is a phenylacetic acid derivative, on IOP after cataract surgery was compared to that of $0.1 \%$ betamethasone sodium phosphate (Rindelon ${ }^{\circledR}$, Shionogi Co. Ltd., Osaka, Japan), which is a topical corticosteroid.

After the next-day examination (defined as day 1), topical administration of an antiinflammatory drug was started; $0.1 \%$ diclofenac and $0.3 \%$ gatifloxacin were topically applied 4 times/day to the left eye, and $0.1 \%$ betamethasone and $0.3 \%$ gatifloxacin were topically applied 4 times/day to the right eye. Drug applications were continued for at least 8 weeks after the surgery.

Total surgery time (minutes) was recorded in each case. The sonication time (seconds) and mean phaco power (percentage) were also noted from the phaco unit's display. The sonication time was defined as the time the foot pedal remained in position 3 . The mean phaco power was defined as the power used during sonication time. The effective phaco time (EPT) was calculated using the following formula: $\mathrm{EPT}=$ sonication time $\mathrm{X}$ mean phaco power/100.

IOP and best corrected logMAR visual acuity (BCVA) in each eye were measured preoperatively and postoperatively at 3 and 7 days, and at 4 and 8 weeks after cataract surgery. The IOP data were obtained using pneumotonometry.

\section{Statistical analysis}

The data are presented as mean \pm standard deviation. Statistical differences between follow-up points of the clinical course in the same eyes were assessed using the Wilcoxon signed-rank test, and the differences between the right and 
left eyes were assessed using the Mann Whitney $U$ test (SPSS ver. 11; SPSS Inc., Chicago, IL, USA).

To clarify the change in IOP, delta IOP $(\Delta \mathrm{IOP})$, defined as a subtraction in IOP at 8 weeks postoperatively from IOP at the preoperative exam, was calculated in each eye. To investigate the correlation between $\triangle \mathrm{IOP}$ and clinical parameters, Pearson's correlation coefficient $(r)$, and a $p$ value were calculated. Since the number of samples was $50, a|r|$ value of more than 0.3 was considered a statistically significant correlation. In all cases, $p<0.05$ was considered to be significant.

To clarify which parameters contribute to $\Delta \mathrm{IOP}$, multiple regression analysis was performed. From multiple regression analysis, a standard partial regression coefficient $(b)$ and a $p$ value in each clinical factor were calculated. Each amount of $|b|$ value reflects a degree of contribution for each factor to the prognosis. To confirm the "goodness-of-fit" in each multiple regression equation, analysis of variance (ANOVA) was performed and a $p$ value of less than 0.05 was considered a good fit.

\section{Results}

The ages of the patients ranged from 60 to 83 years with a mean of $74.8 \pm 5.4$ years. Twenty seven of 50 patients underwent cataract surgery in the right eye first, and then 1 week later in the other eye, while the remaining 23 patients underwent cataract surgery first in the left eye and then in the right eye. The surgery was performed by a single surgeon (MS) and took less than 15 minutes, and no complications occurred.

\section{Alteration of BCVA before and after the cataract surgery}

Before cataract surgery, BCVA in the betamethasone-treated (right) eye was $\log$ MAR $0.348 \pm 0.222$, and BCVA in the diclofenac-treated (left) eye was $\log$ MAR $0.363 \pm 0.233$, thus showing no statistically significant difference in BCVA ( $p=0.785)$. After the surgery, BCVA in all eyes improved, and at the final period of 8 weeks after surgery, BCVA in the right eye was $\log \mathrm{MAR}-0.026 \pm 0.056$, and in the left eye $\log$ MAR $-0.028 \pm 0.057$, so that no statistically significant difference existed between the two groups of eyes 1.992).

\section{Change in IOP after topical administration of antiinflammatory drugs}

Before cataract surgery, IOP in the betamethasone-treated (right) eye was $14.9 \pm 3.2 \mathrm{mmHg}$, and IOP in the diclofenac-treated (left) eye was $15.0 \pm 3.2 \mathrm{mmHg}$, thus showing no statistically significant difference between these two groups $(p=0.986)$.

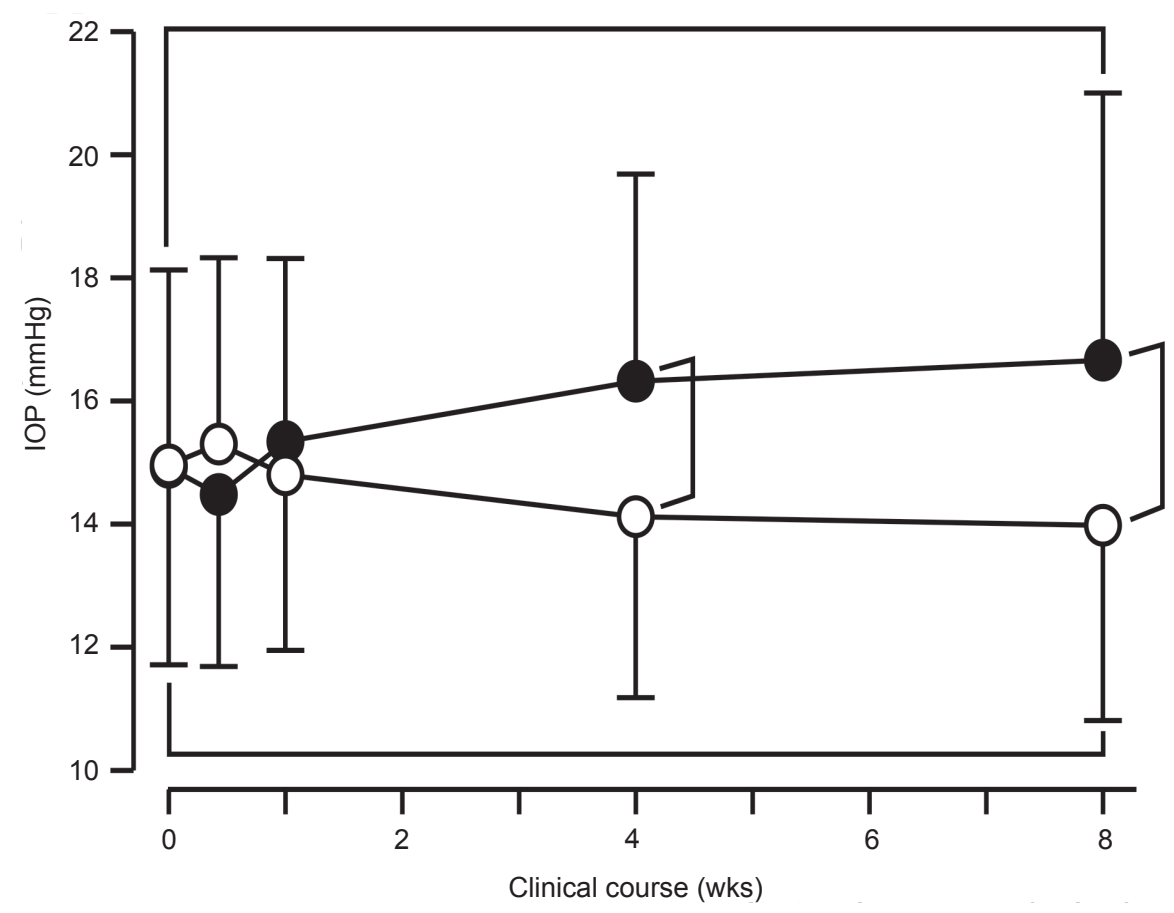

Figure I Clinical course in IOP after the cataract surgery in eyes with postoperative topical administration of betamethasone (closed circle) and that of diclofenac (open circle). The vertical bar indicates standard deviation of the mean. After 4 postoperative weeks, IOP in the betamethasone-treated eye showed a significant increase with compared to that in diclofenac-treated eye. The asterisk $(*)$ indicates statistical significance $(p<0.05)$. 
As shown in Figure 1, IOP in the betamethasone-treated eyes gradually increased to $16.7 \pm 4.3 \mathrm{mmHg}$ ( $p=0.001)$. In contrast, IOP in the diclofenac-treated eyes gradually decreased to $14.0 \pm 3.2 \mathrm{mmHg}(p=0.01)$. There was a statistically significant difference in IOP between each of these groups at $4(p<0.001)$ and 8 weeks $(p<0.001)$.

\section{Relationship between $\Delta \mathrm{IOP}$ and total surgery time}

The total surgery time was $8.58 \pm 1.28 \mathrm{~min}$ in the betamethasone-treated (right) eyes, and $8.74 \pm 1.29 \mathrm{~min}$ in the diclofenac-treated (left) eyes, which shows no statistically significant difference between either group of eyes $(p=0.472)$. To investigate the correlation between $\triangle \mathrm{IOP}$ and total surgery time, Pearson's correlation coefficient was calculated from each plot (Figure 2). $\triangle \mathrm{IOP}$ in the betamethasone-treated eyes was closely correlated with the total surgery time (Figure 2A; $r=0.499, p=0.002$ ); in contrast, $\Delta \mathrm{IOP}$ in the diclofenactreated eyes showed no correlation with the total surgery time (Figure 2B; $r=0.044, p=0.763$ ).

\section{Relationship between $\triangle \mathrm{IOP}$ and EPT}

The EPT was $52.7 \pm 16.6 \mathrm{sec}$ in the betamethasone-treated (right) eyes, and $53.5 \pm 14.9 \mathrm{sec}$ in the diclofenac-treated (left) eyes, which shows no statistically significant difference between both groups of eyes ( $p=0.206)$. To investigate the correlation between $\triangle \mathrm{IOP}$ and the EPT, Pearson's correlation coefficient was calculated from each plot (Figure 3). $\Delta \mathrm{IOP}$ in the betamethasone-treated eyes was closely correlated with the EPT $(r=0.535, p<0.001)$; in contrast, the $\Delta \mathrm{IOP}$ in the diclofenac-treated eyes showed no correlation with the EPT $(r=-0.080, p=0.584)$.

\section{Contribution of clinical factors to $\triangle I O P$ after cataract surgery}

To investigate which clinical factors contributed to the $\Delta \mathrm{IOP}$, multiple regression analysis was performed. The clinical factors were age, gender, preoperative IOP, preoperative BCVA, total surgery time, and EPT. In the betamethasonetreated (right) eyes, $\Delta \mathrm{IOP}$ was $+1.74 \pm 3.41 \mathrm{mmHg}$, and the standard partial regression $|b|$ of total surgery time ( $p=0.019)$ and EPT $(p=0.002)$ was significantly higher than the other factors, indicating that surgery time and EPT contribute to an increase in IOP. The exact probability of this multiple regression equation was confirmed by analysis of variance (ANOVA; $p=0.0001$ ) (Table 1). In contrast, in the diclofenac-treated eyes, $\Delta \mathrm{IOP}$ was $-0.98 \pm 1.89 \mathrm{mmHg}$,
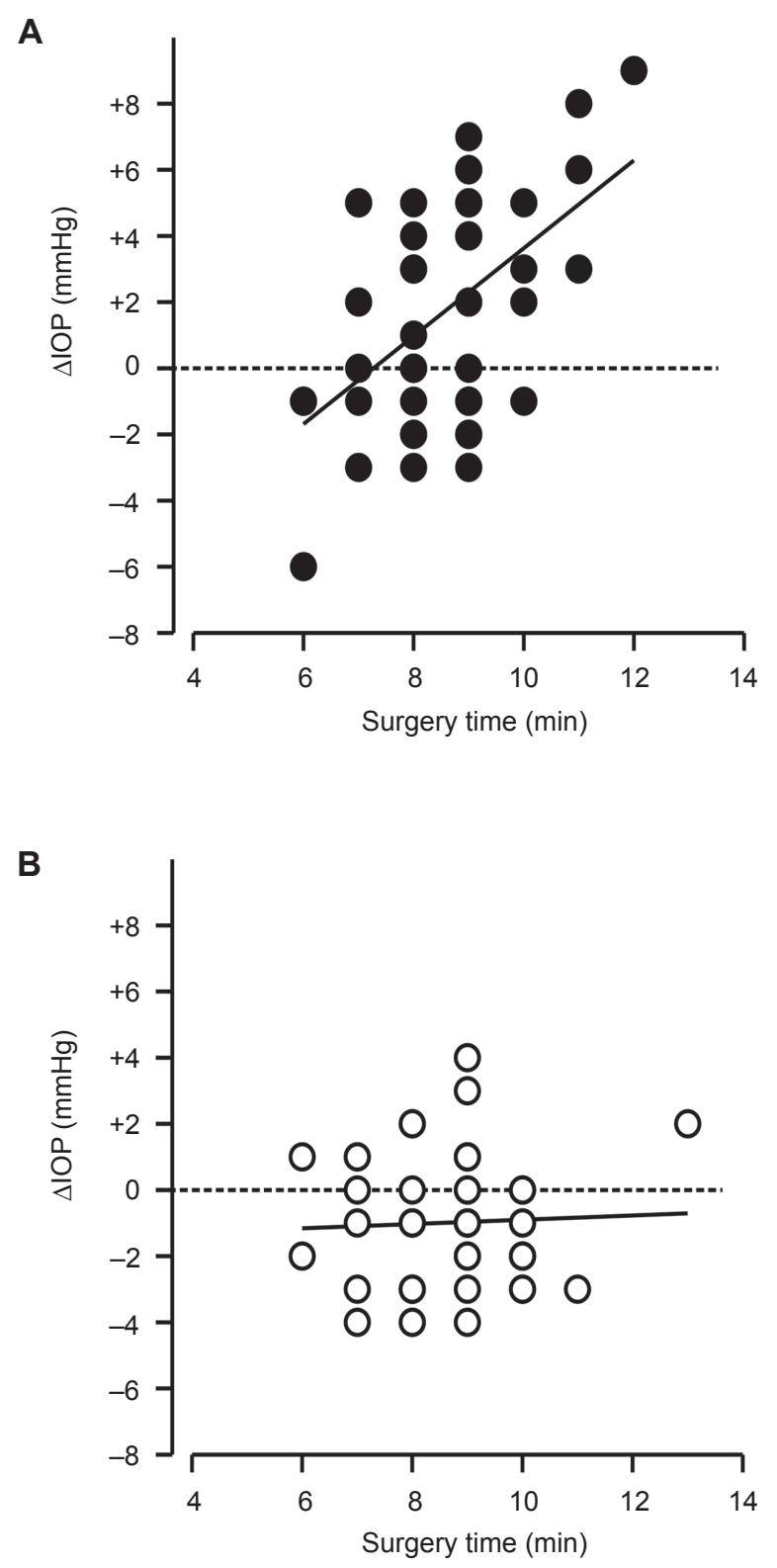

Figure 2 Relationship between the $\triangle \mathrm{IOP}$ and total surgery time in uneventful cataract surgery in postoperative betamethasone-treated eyes $(\mathbf{A})$ and diclofenac-treated eyes (B) Strong correlation was observed in betamethasone-treated eye $(r=0.499$, $p=0.002)$, in contrast little correlation was seen in diclofenac-treated eyes $(r=0.044$, $p=0.763)$.

and there was no statistically significant amount of $|b|$; therefore, a decrease in IOP was not caused by any clinical factors (Table 2).

\section{Discussion}

This prospective case-control study is unique because each patient had one eye treated with betamethasone and the other eye treated with diclofenac after cataract surgery. 
A

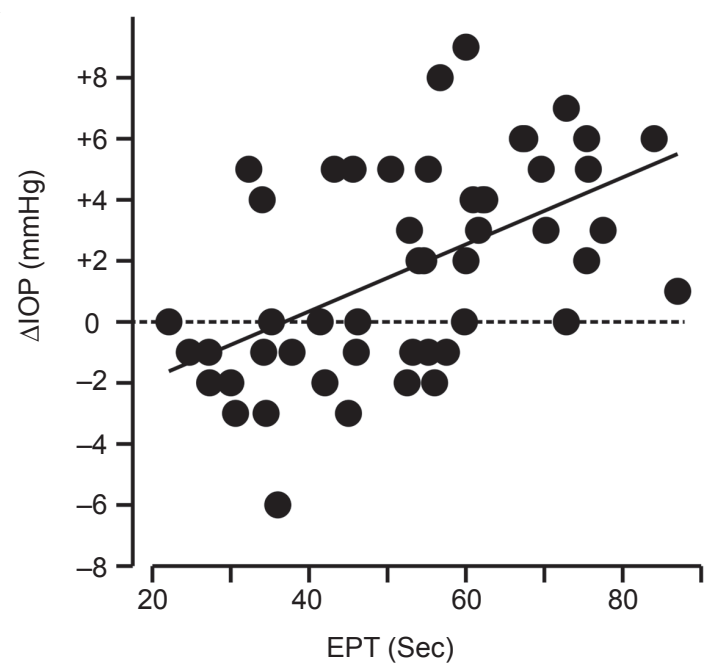

B

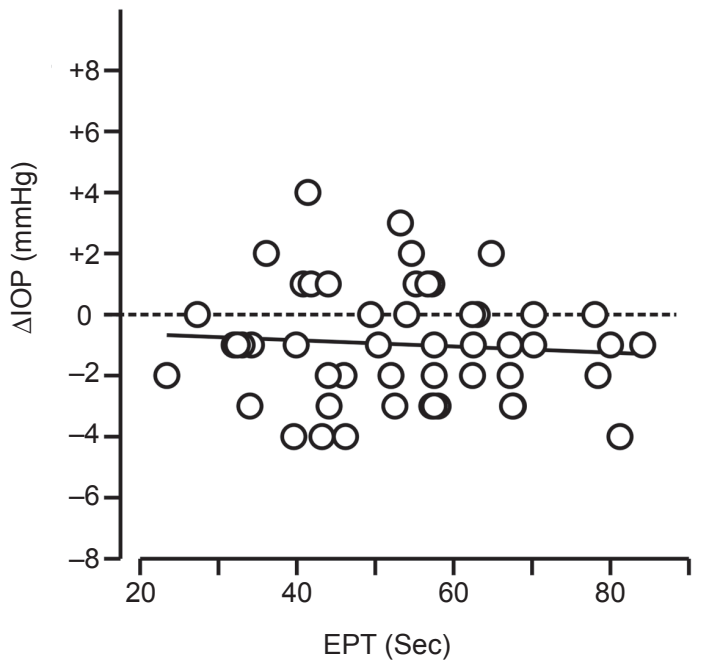

Figure 3 Relationship between the $\triangle I O P$ and EPT in uneventful cataract surgery in postoperative betamethasone-treated eyes $(\mathbf{A})$ and diclofenac-treated eyes (B) Strong correlation was observed in betamethasone-treated eye ( $r=0.535$, $p<0.00 \mathrm{I})$, in contrast little correlation was seen in diclofenac-treated eyes $(r=-0.080$, $p=0.584)$.

In these cases, where patients had both eyes treated, systemic conditions can be excluded.

After uneventful cataract surgery in a normal patient, the major findings of this study are as follows. 1) postoperative IOP was slightly increased in eyes treated with betamethasone, which was dependent upon total surgery time and EPT. 2) In contrast, postoperative IOP in eyes treated with diclofenac was slightly decreased, and was not affected by surgery time or EPT.

According to a recent study, IOP in the nonglaucomatous eye is reduced following uneventful cataract surgery. ${ }^{11}$ In our study, IOP in the diclofenac-treated eyes also showed a significant reduction after cataract surgery. In contrast, IOP in the betamethasone-treated eyes showed significant increase. Interestingly, within 1 week after the surgery, there was no significant difference in IOP between the two groups; however, at 4 and 8 weeks postoperatively, IOP in the corticosteroid (betamethasone)treated eyes was significantly higher than that in NSAID (diclofenac)-treated eyes. It is well known that topical corticosteroid-induced IOP elevation is observed after 3 weeks of administration. ${ }^{12}$ Therefore, IOP elevation after surgery in the betamethasone-treated eyes is possibly "steroid-induced ocular hypertension."

Although the pathogenesis of steroid-induced ocular hypertension still remains unknown, corticosteroid affects cytoskeleton, an extracellular matrix in trabecular meshwork cells, ${ }^{13}$ leading to an increase in the outflow resistance of aqueous humor. However, corticosteroids did not always induce ocular hypertension in all cases. Previous reports indicated $69 \%$ of normal eyes had IOP elevations of at least $5 \mathrm{mmHg}$ and were classified as steroid responders. ${ }^{14}$ In this study 18 of 50 eyes showed more than $5 \mathrm{mmHg}$ of $\triangle \mathrm{IOP}$; in contrast, 23 eyes showed less than $0 \mathrm{mmHg}$ of $\Delta \mathrm{IOP}$, therefore, those 18 patients were considered steroid responders. In these 18 patients, $\Delta \mathrm{IOP}$ in the other (diclofenac-treated) eyes showed less than $2 \mathrm{mmHg}$ (mean $-0.67 \pm 2.0 \mathrm{mmHg}$ ); therefore, diclofenac did not induce ocular hypertension even in steroid responders.

The most interesting result in this study is that an increase of $\triangle \mathrm{IOP}$ after uneventful cataract surgery in betamethasone- treated eyes was related to surgery time and EPT. Longer surgery time and/or EPT usually caused more postoperative inflammation. Generally, it takes more time and/or sonication power to perform cataract surgery in complicated cases including those of patients with a hard nucleus and/or small pupils. Also, beginning surgeons and older patients need more time for surgery. In these cases, diclofenac seems to have some advantages compared with betamethasone for preventing postoperative increases in IOP.

\section{Acknowledgments}

The authors thank Dr Norio Sugimoto in Theranostic Instruments Research Laboratories for his technical support of statistical analyses and useful comments.

\section{Disclosure}

The authors have no competing financial interests in this work. 
Table I Multiple regression analysis for alteration of $\Delta \mathrm{IOP}$ in betamethasone-treated eyes

\begin{tabular}{|c|c|c|c|c|}
\hline \multicolumn{3}{|c|}{ Multiple regression analysis $(n=50)$} & \multicolumn{2}{|c|}{ Dependent $(y)=\Delta I O P(m m H g)$} \\
\hline \multicolumn{3}{|c|}{ Betamethasone } & mean & SD \\
\hline & & & +1.74 & 3.41 \\
\hline \multicolumn{3}{|l|}{ Independent (x) } & \multicolumn{2}{|c|}{ Regression factor } \\
\hline & mean & SD & b & $p$ \\
\hline Age (years) & 74.8 & 5.40 & 0.174 & 0.174 \\
\hline Gender (0:M, I:F) & 0.58 & 0.50 & -0.095 & 0.423 \\
\hline Preoperative IOP $(\mathrm{mmHg})$ & 14.92 & 3.21 & -0.052 & $0.66 \mathrm{I}$ \\
\hline Preoperative BCVA & 0.35 & 0.22 & -0.026 & 0.823 \\
\hline Total surgery time (min) & 8.58 & 1.28 & 0.312 & 0.019 \\
\hline EPT (sec) & 52.70 & 16.60 & 0.399 & 0.002 \\
\hline
\end{tabular}

Notes: $b$ : standard partial regression coefficient calculated from multiple regression equation, $p$ : level of significance.

Abbreviations: BCVA, best corrected visual acuity; EPT, effective phaco time; IOP, intraocular pressure; SD, standard deviation.

ANOVA table from multiple regression equation of $y=$ improvement of VA

\begin{tabular}{lllll}
\hline Cause & SS & $\varphi$ & Ms & F \\
\hline Regression & 260.84 & 6 & 43.47 & 0.001 \\
Residual & 308.78 & 43 & 7.18 & \\
Total & 596.62 & 49 & & \\
\hline
\end{tabular}

Abbreviations: $S S$, sum of square, $\varphi$, degree of freedom; Ms, mean of square; $F$, Ms regression/Ms residual; $p$, exact possibility.

Table 2 Multiple regression analysis for alteration of $\Delta \mathrm{lOP}$ in diclofenac-treated eyes

\begin{tabular}{|c|c|c|c|c|}
\hline \multirow{2}{*}{\multicolumn{2}{|c|}{$\begin{array}{l}\text { Multiple regression analysis }(n=50) \\
\text { Diclofenac }\end{array}$}} & \multirow[b]{5}{*}{ SD } & \multicolumn{2}{|c|}{ Dependent $(y)=\Delta I O P(\mathrm{mmHg})$} \\
\hline & & & mean & SD \\
\hline & \multirow[b]{3}{*}{ mean } & & -0.98 & 1.89 \\
\hline \multirow[t]{2}{*}{ Independent (x) } & & & \multicolumn{2}{|c|}{ Regression factor } \\
\hline & & & $b$ & $p$ \\
\hline Age (years) & 74.8 & 5.40 & 0.046 & 0.755 \\
\hline Gender (0:M, I:F) & 0.58 & 0.50 & 0.079 & 0.581 \\
\hline Preoperative IOP $(\mathrm{mmHg})$ & 14.96 & 3.15 & -0.229 & 0.121 \\
\hline Preoperative BCVA & 0.36 & 0.23 & -0.240 & 0.109 \\
\hline Total surgery time (min) & 8.74 & 1.29 & 0.128 & 0.845 \\
\hline EPT (sec) & 53.46 & 14.87 & -0.120 & 0.414 \\
\hline
\end{tabular}

Notes: $b$ : standard partial regression coefficient calculated from multiple regression equation, $p$ : level of significance.

Abbreviations: BCVA, best corrected visual acuity; EPT, effective phaco time; IOP, intraocular pressure; SD, standard deviation.

ANOVA table from multiple regression equation of $y=$ improvement of VA

\begin{tabular}{lllll}
\hline Cause & SS & $\varphi$ & Ms & $\boldsymbol{F}$ \\
\hline Regression & 27.13 & 6 & 4.52 & 0.271 \\
Residual & 147.85 & 43 & 3.44 & \\
Total & 174.98 & 49 & & \\
\hline
\end{tabular}

Abbreviations: SS, sum of square; $\varphi$, degree of freedom; Ms, mean of square; $F$, Ms regression/Ms residual; $p$, exact possibility. 


\section{References}

1. Rowen S. Preoperative and postoperative medications used for cataract surgery. Curr Opin Ophthalmol. 1999;10:29-35.

2. Nelson ML, Martidis A. Managing cystoid macular edema after cataract surgery. Curr Opin Ophthalmol. 2003;14:39-43.

3. Kersey JP, Broadway DC. Corticosteroid-induced glaucoma: a review of the literatire. Eye. 2006;20:407-16.

4. Jones R 3rd, Rhee DJ. Corticosteroid-induced ocular hypertension and glaucoma: a brief review and update of the literature. Curr Opin Ophthalmol. 2006;17:163-7.

5. Goa KL, Chrisp P. Ocular diclofenac: A review of its pharmacology and clinical use in cataract surgery, and potential in other inflammatory ocular conditions. Drugs and Aging. 1992;2:473-86.

6. Araie M, Sawa M, Takase M. Topical flurbiprofen and diclofenac suppress blood-aqueous barrier breakdown in cataract surgery. Jpn J Ophthalmol. 1983;27:535-42.

7. Flach AJ, Kraff MC, Sanders DR, et al. The quantitative effect of $0.5 \%$ ketorolac tromethamine solution and $0.1 \%$ dexamethasone sodium phosphate solution on postsurgical blood aqueous barrier. Arch Ophthalmol. 1988;106:480-3.
8. Othenin-Girard P, Tritten JJ, Pittet N, et al. Dexamethasone versus diclofenac sodium eyedrops to treat inflammation after cataract surgery. J Cataract Refract Surg. 1994;20:9-12.

9. Strelow SA, Sherwood MB, Broncato LJ, et al. The effect of diclofenac sopdium ophthalmic solution on intraocular pressure following cataract extraction. Ophthalmic Surg. 1992;23:170-5.

10. Emery JM, McIntyre DJ. Patient Selection in Extracapsular Cataract Surgery. St Louis, MO: Mosby-Year Book, Inc. 1983; p. 95-100.

11. Issa SA, Pacheco J, Mahmood U, et al. A novel index for predicting intraocular pressure reduction following cataract surgery. $\mathrm{Br} J$ Ophthalmol. 2005;89:543-6.

12. Gerometta R, Podos SM, Candia OA, et al. Steroid-induced ocular hypertension in normal cattle. Arch Ophthalmol. 2004;122:1492-7.

13. Wordinger RJ, Clark AF. Effects of glucocorticoids on the trabecular meshwork: towards a bettter understanding of glaucoma. Prog Retin Eye Res. 1999;18:629-67.

14. Bartlett JD, Woolley TW, Adams CM. Identification of high intraocular pressure responders to topical ophthalmic corticosteroids. J Ocular Pharmacol. 1993;9:35-45. 
